\title{
The EDGI new take on EDGE: EUS-directed transgastric intervention (EDGI), other than ERCP, for Roux-en-Y gastric bypass anatomy: a multicenter study
}

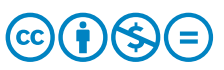

\author{
Authors \\ Shayan S. Irani ${ }^{4}$, John Y. Nasr ${ }^{1}$ \\ Institutions \\ 1 Section of Digestive Diseases, West Virginia University \\ Medicine, Morgantown, West Virginia, United States \\ 2 Division of Gastroenterology and Hepatology, University \\ of North Carolina Medical Center, Chapel Hill, North \\ Carolina, United States \\ 3 Division of Gastroenterology and Hepatology, Johns \\ Hopkins Medical Institutions, Baltimore, Maryland, \\ United States \\ 4 Division of Gastroenterology and Hepatology, Virginia \\ Mason Medical Center, Seattle, Washington, United \\ States
}

Matthew R. Krafft ${ }^{1}$, William Hsueh ${ }^{1}$, Theodore W. James ${ }^{2}$, Thomas M. Runge ${ }^{3}$, Todd H. Baron ${ }^{2}$, Mouen A. Khashab ${ }^{3}$,

submitted 25.3.2019

accepted after revision 25.4.2019

Bibliography

DOI https://doi.org/10.1055/a-0915-2192 |

Endoscopy International Open 2019; 07: E1231-E1240

(c) Georg Thieme Verlag KG Stuttgart · New York

eISSN 2196-9736

Corresponding author

Dr. Matthew R. Krafft, PO Box 9161, One Medical Center

Drive, Morgantown, WV 26506-9161

Fax: +1-304-293-2135

mrkrafft@hsc.wvu.edu

\section{ABSTRACT}

Background and study aims Indications for accessing the duodenum, and/or excluded stomach in Roux-en-Y gastric bypass (RYGB) patients extend beyond diagnosis and treatment of pancreaticobiliary maladies. Given the high technical and clinical success of EUS-directed transgastric ERCP (EDGE) in RYGB anatomy, we adopted this transgastric (anterograde) approach to evaluate and treat luminal and extraluminal pathology in and around the excluded gut in RYGB patients. EUS-directed transgastric intervention ("EDGI"), other than ERCP, is the terminology we have chosen to describe this heterogenous group of transgastric diagnostic and/or interventional endoscopic procedures (transgastric interventions) performed via a lumen-apposing mental stent (LAMS) in select patients with RYGB.

Patients and methods A multicenter $(n=4)$, retrospective study of RYGB patients with suspected luminal or extraluminal pathology, in or around the duodenum and/or excluded stomach, underwent EDGI using LAMS between December 2015 and January 2019.

Results A total of 14 patients ( $78.6 \%$ women; mean age, $55.7+12.4$ years) underwent EDGI via LAMS. Technical and clinical success rates of EDGI were $100 \%$. The most common transgastric interventions were diagnostic EUS of extraluminal pathology $(n=6,42.7 \%)$ and endoscopic biopsy of gastroduodenal luminal abnormalities $(n=5,35.7 \%)$. Two moderate-severity adverse events due to LAMS maldeployment occurred during EUS-JG creation (14.3\%), and each instance was successfully rescued with a bridging stent.

Conclusions A variety of gastroduodenal luminal and extraluminal disorders in RYGB patients can be effectively diagnosed and managed using EDGI via LAMS.

\section{Introduction}

Rising incidence of obesity in the United States has led to increased utilization of bariatric surgery as a means for weight reduction [1]. Roux-en-Y gastric bypass (RYGB), the gold standard of bariatric surgery, restricts both gastric accommodation and absorption of excess nutrients to improve body weight and obesity-related comorbidities [2]. The anatomical configuration of RYGB challenges endoscopists seeking to access the pancreaticobiliary (PB) limb and the excluded stomach. The high incidence of gallstone disease, which can accompany rapid weight loss following RYGB, has driven an evolution of endoscopic procedures that facilitate endoscopic retrograde cholangiopancreatography (ERCP). Notable developments include 


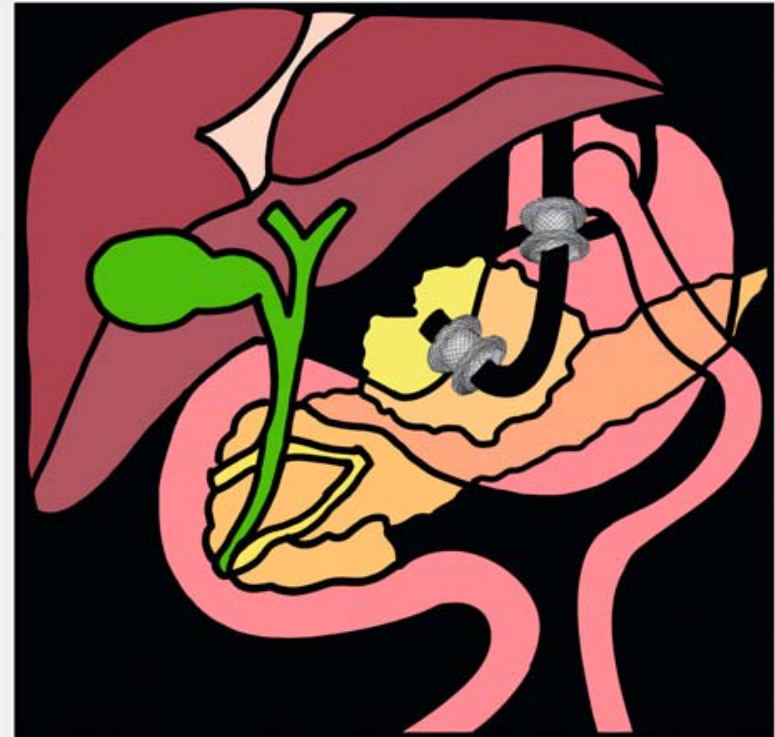

- Fig. 1 GRAPHICAL ABSTRACT Illustrated depiction of EUS-directed transgastric intervention (EDGI) for management of walledoff necrosis (WON) in Roux-en-Y gastric bypass anatomy. Endoscopic access to the gastric remnant is provided by way of a gastrogastric fistula created via a lumen-apposing metal stent (LAMS). A second LAMS is used to drain the WON through the gastric remnant.

ERCP via surgical gastrostomy (i. e., laparoscopy-assisted ERCP) [3], enteroscopy-assisted ERCP (EA-ERCP), anterograde ERCP via percutaneous-assisted transprosthetic endoscopic therapy (PATENT) [4], external EUS-directed transgastric ERCP (external $\operatorname{EDGE})[5,6]$, and most recently, internal $\operatorname{EDGE}[7,8]$. The advent of the lumen-apposing metal stent (LAMS) (AXIOS stent and delivery system, Boston Scientific, Marlborough, Massachusetts, United States) facilitated inception of internal EDGE (herein, referred to as EDGE). The biflanged design of the LAMS creates a stable internal conduit into the excluded stomach for anterograde ERCP, obviating the need for surgical, enteroscope-assisted, and/or percutaneous assistance to perform ERCP.

Indications for pursuing endoscopic access of the excluded gut are far from limited to PB duct pathology. A wide variety of luminal and extraluminal diseases can occur in and around the duodenum and gastric remnant. For this reason, we have begun utilizing LAMS to facilitate diagnostic and/or interventional procedures, other than ERCP, in the duodenum and the excluded stomach of RYGB patients. EUS-directed transgastric intervention (EDGI) is the umbrella term that we are using to collectively describe this myriad of (non-ERCP) transgastric procedures ( $\triangleright$ Fig. 1 ). The aim of our retrospective multicenter study was to evaluate use of EUS-directed transgastric intervention (EDGI), other than ERCP, in RYGB patients.

\section{Patients and methods}

We conducted a multicenter, retrospective study of patients with RYGB anatomy who underwent EUS-directed transgastric intervention (EDGI), other than ERCP, at four tertiary care centers (United States) between December 2015 and January 2019. We defined EDGI as EUS-directed gastrogastrostomy (or jejunogastrostomy) creation via LAMS, followed by a transgastric diagnostic and/or interventional endoscopic procedure (herein, referred to as a transgastric intervention), other than ERCP, within the gastric remnant or duodenum. EDGI was performed as either a primary or secondary procedure (i. e., EDGI was considered a secondary procedure if preceded by a failed alternative modality, such as overtube-assisted enteroscopy). EDGI was performed in single or dual endoscopic sessions. Transgastric interventional techniques were performed at the discretion of each participating advanced endoscopist. Within the EDGI study population, four patients underwent EDGE (either prior to EDGI or concurrently with EDGI), and were previously reported in a single-center study [9]. This study was approved by the Institutional Review Boards for Human Research and complied with Health Insurance Portability and Accountability Act regulations at each participating institution.

\section{Technical success, clinical success, and other collected data}

Data collection was largely categorized according to the two principal stages of EDGI: EUS-GG or JG creation and the intended transgastric intervention. Technical success of EUS-directed gastrogastrostomy/jejunogastrostomy creation (herein, referred to as EUS-GG or JG, which is a step within EDGI) was defined as successful transmural fistula creation using LAMS. Technical success of the transgastric intervention was defined as completion of the diagnostic and/or interventional endoscopic procedure by way of the endoscopically-created transmural fistula. Clinical success of the transgastric intervention was defined as attainment of diagnosis and/or partial or complete symptom relief. Adverse events (AEs) associated with either EUS-GG or the transgastric intervention were recorded. The American Society for Gastrointestinal Endoscopy (ASGE) lexicon for endoscopic AEs was used to classify AE severity [10].

\section{Procedural descriptions}

EUS-directed gastrogastrostomy/jejunogastrostomy creation (EUS-GG)

All procedures were performed under general anesthesia with endotracheal intubation and under fluoroscopy. A standard therapeutic channel oblique linear echoendoscope (GFUCT180; Olympus, Central Valley, Pennsylvania, United States) was introduced into the gastric pouch, or jejunum (Roux limb), to scan for the gastric remnant. The closest area (i. e., puncture point) between the gastric pouch and the gastric remnant was located. A puncture point between the jejunum (Roux limb) and gastric remnant was sought if a gastro-gastric puncture point was not identifiable. Color Doppler was used to identify and avoid intervening vascular structures. An endoscopic ultra- 
sound 19-gauge aspiration needle (Expect Slimline; Boston Scientific, Marlborough, Massachusetts, United States) was passed through the echoendoscope working channel, and EUS-directed transmural puncture of the gastric remnant was performed. The stylet was withdrawn from the needle and watersoluble contrast medium was injected into the gastric remnant to confirm needle placement under fluoroscopy. At least $100 \mathrm{~mL}$ of contrast medium and sterile water was injected to distend the gastric remnant, under endosonographic and fluoroscopic visualization.

LAMS device specifics and deployment technique varied by endoscopist. Either an electrocautery-enhanced (ECE) or a non-cautery enhanced (non-ECE) LAMS ("Hot" or "Cold" AXIOS stent and delivery system; Boston Scientific, Marlborough, Massachusetts, United States) was deployed into the gastric remnant via freehand or over-the-guidewire form. Lumen diameter of the LAMS was either $20 \mathrm{~mm}$ or $15 \mathrm{~mm}$. The distal flange of the LAMS was deployed into the gastric remnant under endosonographic and/or fluoroscopic guidance. The proximal flange was deployed into the gastric pouch or jejunum via direct endoscopic visualization. In one case, the proximal flange of the LAMS was anchored to the mucosa, using endoscopic suturing (Overstitch; Apollo Endosurgery, Austin, Texas, United States), to mitigate risk of stent dislodgment during scope passage. In a separate case, a 20-mm $\times 150-\mathrm{mm}$ esophageal fully-covered self-expanding metal stent (Niti-S through-the-scope esophageal stent; Taewoong Medical, Seoul, Korea) was deployed through an acutely angled 20-mm JG LAMS to redirect the angle of the indwelling LAMS so that a therapeutic linear echoendoscope could be successfully passed into the gastric remnant ( $\triangleright$ Fig. 2c). In select cases, the LAMS was serially dilated using a through-the-scope (TTS) balloon dilator (controlled radial expansion balloon dilatation catheter; Boston Scientific, Marlborough, Massachusetts, United States) to facilitate endoscopic traversal of the LAMS.

\section{Index transgastric diagnostic and/or interventional endoscopic procedures (transgastric interventions)}

A variety of transgastric interventions were performed for suspected luminal and/or extraluminal pathology. The index transgastric intervention was performed either during the same session as EUS-GG creation (i. e., single-session EDGI) or in a subsequent session after EUS-GG creation (i.e., dual-session EDGI). Depending on the indication for EDGI, either a diagnostic gastroscope (GIF-HQ190; Olympus, Central Valley, Pennsylvania, United States), therapeutic gastroscope (GIF-1TH190; Olympus) and/or linear echoendoscope (GF-UCT180; Olympus) was passed through the LAMS, or through a mature GG/JG fistula immediately following LAMS removal, into the bypassed stomach. Fluoroscopy was used to help guide the scope in a parallel fashion through the LAMS to reduce risk of LAMS dislodgment. Transgastric EUS was performed in eight of 14 patients (57.1\%) using a 14.6-mm (outside diameter) therapeutic linear echoendoscope. In four cases, the therapeutic echoendoscope was passed through a mature GG/JG fistula immediately following LAMS removal (all dual-session EDGIs). In three cases, transgastric passage of the therapeutic echoendoscope occurred through an indwelling 20-mm LAMS (all dual-session EDGIs). In one case, transgastric passage of the therapeutic echoendoscope occurred through an indwelling 15-mm LAMS, after LAMS fixation (single-session EDGI).

\section{LAMS removal and follow-up}

Once transmural access to the excluded stomach and duodenum was no longer required, the LAMS was removed using either a standard large-diameter polypectomy snare or a grasping forceps (Rat tooth alligator jaw grasping forceps; Olympus). Transmural fistula tract closure was either spontaneous (i.e., stent removal alone) or facilitated by argon plasma coagulation (APC) to the fistula edges to promote re-epithelialization (closure) of the fistula tract. No cases of endoscopic suturing for GG/JG fistula closure occurred. Surveillance for persistent GG/ JG fistula was performed using UGI series, serial weight monitoring, or CT scan with IV and oral contrast.

\section{Statistical Analysis}

Statistical analysis was performed using STATA statistical software version 15.0 (Statacorp, College Station, Texas, United States). Categorical variables are reported as percentages. Quantitative variables are reported either as mean \pm standard deviation or median with interquartile range (IQR).

\section{Results}

A total of 14 RYGB patients (11 females, 3 males) underwent EDGl during the study period. Mean patient age was $55.7 \pm 12.4$ years $(\triangleright$ Table $\mathbf{1}$ ). Indications for EDGI included suspected extraluminal and luminal pathology. Extraluminal indications included pancreas mass on imaging $(n=3,21.4 \%)$, inflammatory pancreatic fluid collections (PFC) $(n=2,14.3 \%)$, suspected cholangiocarcinoma $(n=1,7.1 \%)$, abnormal liver biochemical and function tests $(n=1,7.1 \%)$, common bile duct dilation on imaging in a patient requiring familial pancreatic cancer screening $(n=1,7.1 \%)$, and idiopathic recurrent acute pancreatitis $(n=1,7.1 \%)$. Luminal indications were abnormal gastric imaging $(n=2 ; 14.2 \%)$, duodenal mass $(n=1$, $7.1 \%)$, duodenal stricture $(n=1,7.1 \%)$, and duodenal ulcer perforation ( $n=1,7.1 \%$ ). EDGI was performed as a primary procedure in 13 cases $(92.9 \%)$ and a secondary procedure in 1 case (7.1\%), after technical failure of enteroscopy.

The technical success rate for EUS-GG was $100 \%(n=14)$ ( $>$ Table 2). Transmural fistula routes consisted of eight gastrogastrostomies (57.1\%) and six jejunogastrostomies (42.9\%). EUS-GG was performed using ECE-LAMS in 12 cases (85.7\%) and non-ECE LAMS in two cases (14.3\%). Freehand form was used in 10 cases $(71.4 \%$ ) and over-the-wire (OTW) technique was used in four cases $(28.6 \%)$. LAMS lumen diameter was $20 \mathrm{~mm}$ in eight cases (57.1\%) and $15 \mathrm{~mm}$ in six cases (42.9\%). LAMS fixation (endoscopic suturing) was used in one case (7.1\%) to mitigate risk of LAMS dislodgment during transgastric passage of a therapeutic echoendoscope through a $15-\mathrm{mm}$ LAMS in a single-session EDGI. Re-angulation of an acutely-angled indwelling 20-mm JG LAMS, via placement of a throughthe-LAMS $20-\mathrm{mm} \times 150-\mathrm{mm}$ esophageal FCSEMS, occurred in 

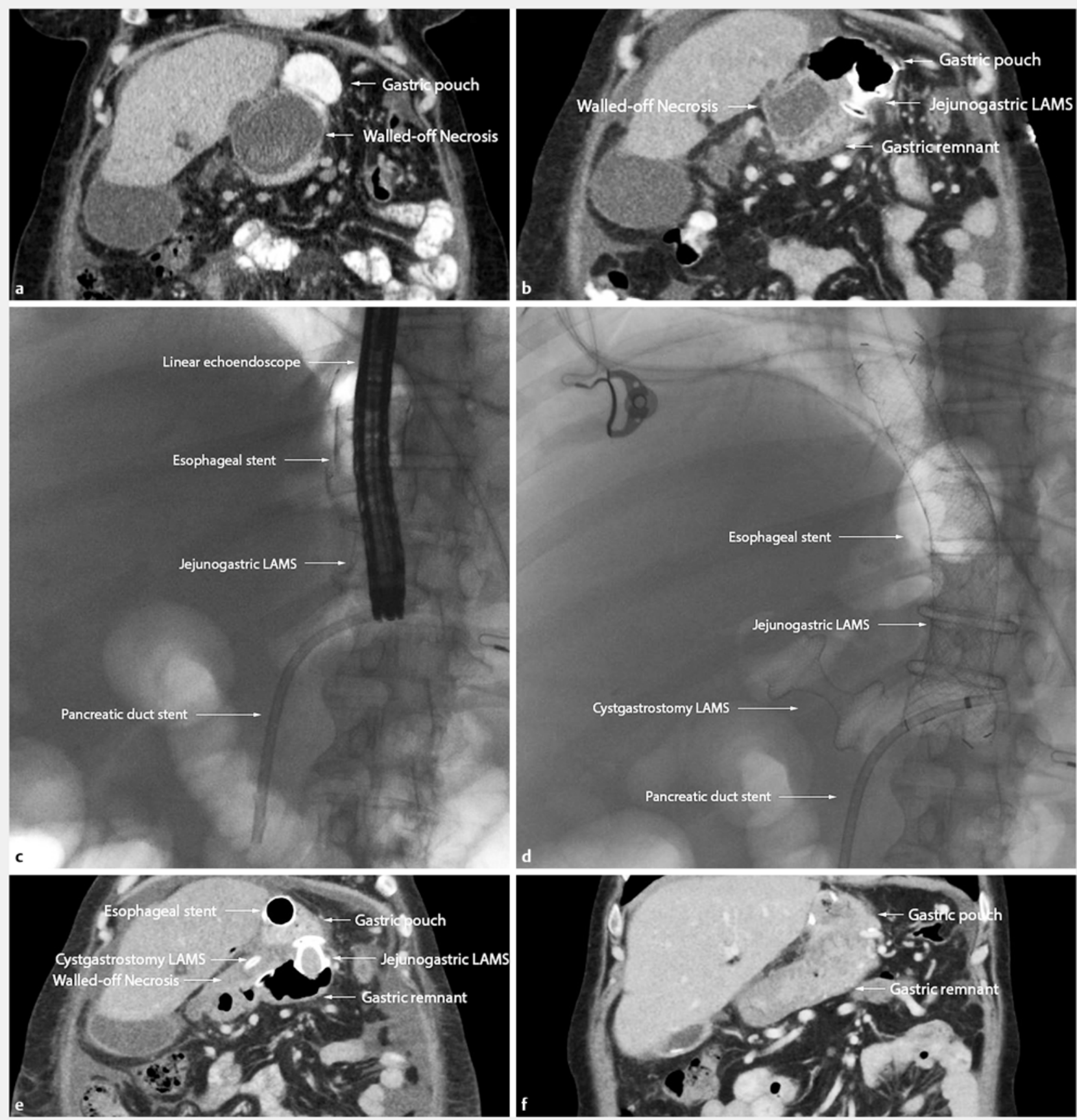

- Fig. 2 a CT abdomen/pelvis (coronal section) demonstrating a pancreatic walled-off necrosis (WON) adjacent to the gastric pouch in a Roux-en-Y gastric bypass (RYGB) patient. b CT abdomen/pelvis (coronal section) demonstrating the pancreatic WON adjacent to the gastric pouch and gastric remnant, after EUS-directed jejunogastric (JG) fistula creation with a 20-mm $\times 10$-mm electrocautery-enhanced lumenapposing metal stent (LAMS) (Hot AXIOS stent and delivery system; Boston Scientific, Marlborough, Massachusetts, United States). c Fluoroscopic image of a linear echoendoscope (GF-UCT180; Olympus, Central Valley, Pennsylvania, United States) inserted through a 20-mm $\times$ 150-mm esophageal fully covered self-expanding metal stent (FCSEMS) (Niti-S through-the-scope esophageal stent; Taewoong Medical, Seoul, Korea) that was placed through the jejunogastric LAMS. The esophageal FCSEMS was placed through the JG 20-mm LAMS to readjust the angle of the LAMS so that the linear echoendoscope could be passed into the gastric remnant without LAMS dislodgement. A previously placed $10-\mathrm{Fr} \times 9-\mathrm{cm}$ straight plastic pancreatic duct stent is visible. $\mathbf{d}$ Fluoroscopic image of a newly deployed $15-\mathrm{mm} \times 10$-mm LAMS between the pancreatic WON and gastric remnant (cystgastrostomy). The esophageal FCSEMS within the JG LAMS, and the pancreatic duct stent, are visible. e CT abdomen/pelvis (coronal section) demonstrating a nearly resolved pancreatic WON, with the LAMS cystgastrostomy deployed between the WON and gastric remnant. The proximal end of the esophageal FCSEMS, through the jejunogastric LAMS, is visible. $\mathbf{f} C T$ abdomen/ pelvis (coronal section) 6-weeks after EUS-directed cystgastrostomy in a patient with a RYGB demonstrating complete resolution of the WON. 
- Table 1 Patient characteristics, EUS-directed transgastric intervention (EDGI) cohort.

\begin{tabular}{|c|c|}
\hline & EDGI $(n=14)$ \\
\hline \multicolumn{2}{|l|}{ Participating centers } \\
\hline - Johns Hopkins Medical Institutions & $2(14.3)$ \\
\hline - University of North Carolina Medical Center & $4(28.6)$ \\
\hline - Virginia Mason Medical Center & $2(14.3)$ \\
\hline - West Virginia University Medicine & $6(42.9)$ \\
\hline Age, years, mean $\pm S D$ & $55.7 \pm 12.4$ \\
\hline \multicolumn{2}{|l|}{ Sex } \\
\hline - Men & $3(21.4)$ \\
\hline - Women & $11(78.6)$ \\
\hline \multicolumn{2}{|l|}{ Indication for EDGI } \\
\hline \multicolumn{2}{|l|}{ - Extraluminal } \\
\hline - Pancreas mass on imaging & $3(21.4)$ \\
\hline - Inflammatory pancreatic fluid collection & $2(14.3)$ \\
\hline - Suspected cholangiocarcinoma & $1(7.1)$ \\
\hline $\begin{array}{l}\text { - Abnormal liver biochemical and function } \\
\text { tests with unremarkable noninvasive ab- } \\
\text { dominal imaging }\end{array}$ & $1(7.1)$ \\
\hline $\begin{array}{l}\text { - Common bile duct dilation on imaging in a } \\
\text { patient requiring familial pancreatic cancer } \\
\text { screening }\end{array}$ & $1(7.1)$ \\
\hline - Idiopathic recurrent acute pancreatitis & $1(7.1)$ \\
\hline \multicolumn{2}{|l|}{ - Luminal } \\
\hline - Abnormal gastric imaging on CT or PET & $2(14.2)$ \\
\hline - Duodenal mass & $1(7.1)$ \\
\hline - Duodenal stricture & $1(7.1)$ \\
\hline - Duodenal ulcer perforation & $1(7.1)$ \\
\hline EDGI performed as primary procedure & $13(92.9)$ \\
\hline EDGI performed as secondary procedure & $1(7.1)$ \\
\hline $\begin{array}{l}\text { Values correspond to n (\%), unless otherwise stated. } \\
\text { EDGI, EUS-directed transgastric intervention; CT, comp } \\
\text { PET, Positron emission tomography; SD, standard devia }\end{array}$ & tomography; \\
\hline
\end{tabular}

one case to accommodate transgastric passage of a therapeutic echoendoscope in Step 2 of a dual-session EDGI (7.1\%). TTSballoon serial dilation of the LAMS was performed prior to scope passage in 8 cases $(57.1 \%)$.

LAMS maldeployment occurred in two separate cases (14.3\%) of OTW deployment of $15-\mathrm{mm}$ ECE-LAMSs during EUS-JG creation. Successful intraprocedural OTW rescue was performed with bridging $18-\mathrm{mm}$ diameter esophageal FCSEMSs (Niti-S through-the-scope esophageal stent, Taewoong Medical, Seoul, Korea), ultimately rendering each EUSJG creation complete. The two cases of LAMS maldeployment were graded moderate-severity AEs because of the need for a
- Table 2 Procedure characteristics, EUS-directed gastrogastrostomy creation (EUS-GG or JG) $(n=14)$.

\begin{tabular}{|c|c|}
\hline Characteristics & Value \\
\hline Technical success & $14(100)$ \\
\hline \multicolumn{2}{|l|}{ Fistula route } \\
\hline - Gastrogastrostomy & $8(57.1)$ \\
\hline - Jejunogastrostomy & $6(42.9)$ \\
\hline \multicolumn{2}{|l|}{ LAMS type } \\
\hline - Electrocautery-enhanced LAMS (Hot) & $12(85.7)$ \\
\hline - Non-cautery enhanced LAMS (Cold) & $2(14.3)$ \\
\hline \multicolumn{2}{|l|}{ LAMS diameter } \\
\hline - 20-mm & $8(57.1)$ \\
\hline - 15-mm & $6(42.9)$ \\
\hline \multicolumn{2}{|l|}{ LAMS deployment technique } \\
\hline " Freehand & $10(71.4)$ \\
\hline - Over-the-wire & $4(28.6)$ \\
\hline $\begin{array}{l}\text { - LAMS fixation (anchoring before transgastric inter- } \\
\text { vention) }\end{array}$ & $1(7.1)$ \\
\hline $\begin{array}{l}\text { - Redirection of LAMS angle via esophageal FCSEMS } \\
\text { (stent within a stent), to facilitate therapeutic linear } \\
\text { echoendoscope passage }\end{array}$ & $1(7.1)$ \\
\hline $\begin{array}{l}\text { " LAMS dilation (TTS-balloon dilation before trans- } \\
\text { gastric intervention) }\end{array}$ & $8(57.1)$ \\
\hline \multicolumn{2}{|l|}{ Adverse events } \\
\hline - LAMS maldeployment & $2(14.3)$ \\
\hline - Rescue via bridging esophageal FCSEMS & $2(100)$ \\
\hline
\end{tabular}

Values correspond to $n(\%)$, unless otherwise stated.

EUS-directed gastrogastrostomy (EUS-GG) refers to both gastrogastrostomy (GG) and jejunogastrostomy (JG) creation (for brevity); LAMS, lumen-apposing metal stent; $\mathrm{mm}$, millimeters; TTS-balloon dilation, through-thescope balloon dilation; SD, standard deviation.

rescue maneuver and unplanned post-procedural hospitalizations. No other EUS-GG-related AEs occurred during the study.

The technical and clinical successes of the index transgastric interventions were $100 \%(n=14)$ ( $\triangleright$ Table 3$)$. Scope types used for transgastric interventions included 1 diagnostic gastroscope $(7.1 \%)$, 5 therapeutic gastroscopes (35.7\%), and 8 therapeutic linear echoendoscopes (57.1\%). Extraluminal interventions completed were diagnostic EUS of the pancreaticobiliary system $(n=3,21.4 \%)$, EUS-guided PFC drainage via ECELAMS $(n=2,14.3 \%)$, EUS-FNB of suspected cholangiocarcinoma ( $n=1,7.1 \%)$, EUS-guided liver biopsy $(n=1,7.1 \%)$, and EUSFNA of pancreatic cystic neoplasm $(n=1,7.1 \%)$. Luminal interventions included gastroduodenal luminal biopsies $(n=5$, $35.7 \%$ ) and closure of a perforated duodenal ulcer using a hemoclip ( $n=1,7.1 \%$ ). No instances of LAMS dislodgement or other AEs occurred during any of the index transgastric interventions. 
- Table 3 Procedure characteristics, index transgastric diagnostic and/ or interventional endoscopic procedures $(n=14)$.

\begin{tabular}{|c|c|}
\hline Characteristics & Value \\
\hline Technical success & $14(100)$ \\
\hline Clinical success & $14(100)$ \\
\hline \multicolumn{2}{|l|}{ Scope type } \\
\hline - Diagnostic gastroscope & $1(7.1)$ \\
\hline - Therapeutic gastroscope & $5(35.7)$ \\
\hline - Linear echoendoscope & $8(57.1)$ \\
\hline \multicolumn{2}{|l|}{ Transgastric endoscopic interventions } \\
\hline \multicolumn{2}{|l|}{ - Extraluminal } \\
\hline $\begin{array}{l}\text { - EUS of pancreaticobiliary system (diagnostic EUS } \\
\text { without biopsy) }\end{array}$ & $3(21.4)$ \\
\hline - EUS-directed PFC drainage via ECE-LAMS & $2(14.3)$ \\
\hline - EUS-FNB of suspected cholangiocarcinoma & $1(7.1)$ \\
\hline - EUS-FNB of liver & $1(7.1)$ \\
\hline - EUS-FNA of pancreatic cystic neoplasm & $1(7.1)$ \\
\hline \multicolumn{2}{|l|}{ - Luminal } \\
\hline - Gastroduodenal luminal biopsy & $5(35.7)$ \\
\hline - Perforated duodenal ulcer closure via hemoclip & $1(7.1)$ \\
\hline LAMS dislodgement during transgastric intervention & 0 \\
\hline Adverse events & 0 \\
\hline \multicolumn{2}{|c|}{$\begin{array}{l}\text { Values correspond to n (\%), unless otherwise stated. } \\
\text { Scope type refers to the endoscope that was used to perform the transgas- } \\
\text { tric intervention; Therapeutic gastroscope (GIF-1TH190, Olympus, Central } \\
\text { Valley, Pennsylvania, United States); Linear echoendoscope (GF-UCT180, } \\
\text { Olympus, Central Valley, Pennsylvania, United States); EUS-directed PFC } \\
\text { drainage via ECE-LAMS, EUS-directed inflammatory pancreatic fluid collec- } \\
\text { tion drainage via electrocautery-enhanced lumen-apposing metal stent; } \\
\text { EUS-FNB, EUS-guided fine-needle biopsy; EUS-FNA, EUS-guided fine-needle } \\
\text { aspiration. }\end{array}$} \\
\hline
\end{tabular}

Single-session EDGI (i. e., EUS-GG plus transgastric intervention) occurred in 5 patients (35.7\%) ( $\triangleright$ Table 4$)$. Only one transgastric EUS was performed as a single-session EDGI. Mean procedure time for single-session EDGI was $49.4 \pm 13$ minutes. Median postprocedure hospitalization time for single-session EDGI was 0.5 days (IQR, $0-1.25$ ). Dual-session EDGI was performed in nine patients (64.3\%) and the median interval between EUSGG and the transgastric intervention was 18 days (IQR, $7-30$ ). Mean procedure time for dual-session EDGI was $84.2 \pm 32.9$ minutes. Median postprocedure hospitalization time for dualsession EDGI was 2.5 days (IQR, $0.75-11.5$ ).

Endoscopic removal of the indwelling LAMS occurred in 11 patients after a median LAMS dwell time of 38 days (IQR, $20-$ 124) ( $\triangleright$ Table 5). Fistula closure occurred by secondary intent (i. e., spontaneous closure) in seven patients (63.6\%) and via application of APC to the fistula edges in three patients (27.3\%). Indefinite fistula patency was maintained in one patient using a DPPS through the mature GGF (9.1\%); this patient gained $20 \mathrm{~kg}$ on 1.5-year follow-up. The remaining three patients were either
Table 4 Procedure intervals, procedure times, post-procedure hospitalization duration $(n=14)$.

\begin{tabular}{|l|c|}
\hline Characteristics & Value \\
\hline Single-session EDGI & $5(35.7)$ \\
\hline $\begin{array}{l}\text { - Procedure time (EUS-GG plus transgastric } \\
\text { intervention), minutes, mean } \pm \text { SD }\end{array}$ & $49.4 \pm 13$ \\
\hline - $\begin{array}{l}\text { Postprocedure hospitalization, days, median } \\
\text { (IQR) }\end{array}$ & $0.5(0-1.25)$ \\
\hline \begin{tabular}{l} 
Dual-session EDGI \\
\hline - Interval between EUS-GG creation and trans- \\
gastric intervention, days, median (IQR)
\end{tabular} & $18(7-30)$ \\
\hline - Procedure time (EUS-GG plus transgastric \\
intervention), minutes, mean \pm SD
\end{tabular}

- Table 5 Procedure characteristics, LAMS removal and GG fistula closure $(n=11)$.

\begin{tabular}{|c|c|}
\hline Characteristics & Value \\
\hline \multicolumn{2}{|l|}{ No. patients with eventual endoscopic LAMS removal } \\
\hline \multicolumn{2}{|l|}{ - Fistula closure timing and method } \\
\hline $\begin{array}{l}\text { - Spontaneous fistula closure at time of LAMS } \\
\text { removal }\end{array}$ & $7(63.6)$ \\
\hline $\begin{array}{l}\text { - Temporary maintenance of fistula patency, } \\
\text { after LAMS removal, via DPPS through GG. APC } \\
\text { later used to facilitate fistula closure. }\end{array}$ & $3(27.3)$ \\
\hline $\begin{array}{l}\text { - Indefinite maintenance of fistula patency, after } \\
\text { LAMS removal, via DPPS through GG. }\end{array}$ & $1(9.1)$ \\
\hline $\begin{array}{l}\text { - LAMS dwell time (interval between EUS-GG and } \\
\text { endoscopic LAMS removal), days, median (IQR) }\end{array}$ & $38(20-124)$ \\
\hline \multicolumn{2}{|c|}{$\begin{array}{l}\text { Values correspond to n (\%), unless otherwise stated. } \\
\text { LAMS, lumen-apposing metal stent; IQR, interquartile range; OTSC, over- } \\
\text { the-scope clip; APC, argon plasma coagulation; DPPS, double-pigtail plastic } \\
\text { stent; GG, gastrogastrostomy. }\end{array}$} \\
\hline
\end{tabular}

lost to follow-up or transferred care to another institution. Long-term follow-up after stent removal (fistula closure) was available for 10 patients. Surveillance for unintentional persistent GG fistula was performed using serial weight measurements $(n=6,60 \%)$, upper gastrointestinal (UGI) series $(n=3$, $30 \%$ ), and computed tomography (CT) scan with intravenous and oral contrast $(n=1,10 \%)$. No unintentional weight gain via serial weight measurements or persistent GGFs were detected radiographically ( $\triangleright$ Table 6$)$. 
Table 6 Follow-up characteristics, monitoring for an unintentional persistent gastrogastric (GG) fistula $(n=10)$.

\begin{tabular}{|l|l|}
\hline Characteristics & Value \\
\hline Monitoring for persistent GG fistula & \\
\hline - Serial weight measurements & $6(60)$ \\
\hline - UGl series & $3(30)$ \\
\hline - CT scan with intravenous and oral contrast & $1(10)$ \\
\hline $\begin{array}{l}\text { Unintentional weight gain and/or persistent GG } \\
\text { fistula }\end{array}$ & 0 \\
\hline $\begin{array}{l}\text { Values correspond to } n \text { (\%), unless otherwise stated. } \\
\text { UGl, upper gastrointestinal series; CT, computed tomography. }\end{array}$ \\
\hline
\end{tabular}

\section{Discussion}

Roux-en-Y gastric bypass (RYGB) is the second most common bariatric surgery performed in the United States. (after sleeve gastrectomy), with an estimated 40,584 cases of RYGB performed in 2017 [11]. Per oral endoscopic examination of the duodenum and gastric remnant has remained a challenge given that the combined lengths of the esophagus $(25 \mathrm{~cm})$, gastric pouch $(5 \mathrm{~cm})$, Roux limb $(75-150 \mathrm{~cm})$, ligament of Treitz to jejunojejunal anastomosis $(30-50 \mathrm{~cm})$, duodenum $(25-38 \mathrm{~cm})$, and the excluded stomach $(10-15 \mathrm{~cm})$ can range from 2 to 3 meters $[12,13]$. The most common indication for obtaining endoscopic access to the PB limb in RYGB patients is gallstonerelated disease (i.e., up to $45 \%$ of patients form gallstones in the months following bariatric surgery) $[14,15]$. EDGE is the culmination of a $20+$-year evolution of endoscopy-based procedures that facilitate ERCP in RYGB anatomy. EDGE is technically and clinically successful because it is performed internally by a single team (i.e., without the need for percutaneous or surgical assistance), it is associated with very high technical success of both EUS-GG creation and pancreaticobiliary cannulation, and its reported $\mathrm{AE}$ rate is similar to EA-ERCP $[16,17]$.

EDGI is an umbrella term that encompasses transgastric (through GG or JG fistula) diagnostic and/or interventional endoscopic procedures, other than ERCP, in the duodenum and/or gastric remnant in RYGB patients. EDGI is a new category of procedures that has not been studied separately before. A small number of transgastric EUS cases have been reported in studies focused on transgastric ERCP $[9,17]$. The first reported EDGls (not referred to as "EDGI" in the original manuscript) were three RYGB patients with pancreatic lesions who underwent EUS-directed transgastric EUS-FNA by way of mature fistula tracts (GG/JG) formed via 15-mm LAMSs [17]. Reports of transgastric procedures, other than transgastric ERCP and transgastric EUS, are not available in the existing literature.

Results of our study show that EDGI can be performed for a variety of indications (other than ERCP), in RGYB anatomy, with outstanding technical (100\%) and clinical success (100\%). The most common forms of EDGI in our patient cohort were transgastric EUS ( \pm FNA or FNB) for diagnosis of extraluminal pathology $(n=6,42.7 \%)$ and transgastric endoscopic biopsy of gastro- duodenal luminal abnormalities ( $n=5,35.7 \%)$. Our study included two novel forms of therapeutic EDGI: transgastric drainage of PFC using ECE-LAMS $(\mathbf{n}=2)(\triangleright$ Fig. $2 \mathbf{a}-\mathbf{f})$ and transgastric closure of a perforated duodenal ulcer using a hemoclip $(n=1)$ ( $\triangleright$ Fig.3a-c). The clinical successes of transgastric PFC drainage and ulcer closure spared these patients from surgery that would have been more complex due to their RYGB anatomy.

EDGI was selected as the primary procedure in almost all of our study patients $(n=13 ; 92.9 \%)$, whereas in a single case, failed enteroscopy preceded EDGI. Overtube-assisted enteroscopy (OAE) is the established means of internally accessing the gastric remnant and duodenum in RYGB patients; however, we believe that EDGI has several distinct advantages compared to OAE. For diagnosis and management of gastroduodenal extraluminal pathology, EDGl is the only internal means because OAE with EUS capabilities does not exist. For diagnosis and management of gastroduodenal luminal pathology, the literature shows a higher rate of technical success for gastroduodenal access via EUS-GG creation compared to enteroscopy. A recent study by Bukhari et al. compared EDGE to enteroscopy-assisted ERCP (EA-ERCP) in RYGB anatomy. The technical success rate was significantly higher in the EDGE versus the EA-ERCP group $(100 \%$ vs $60 \%, P<0.001)$. Among the reasons for the $40 \%$ rate of technical failure in the EA-ERCP group was an inability to reach the papilla in $20 \%$ [16]. In a prospective observational study of elective double-balloon enteroscopy of the excluded stomach in RYGB anatomy by Kuga et al., the excluded stomach was reached in 35/40 patients (i.e., technical success $87.5 \%$ ) [12], which is less than the near $100 \%$ technical success rate reported in the EDGE literature [16-18].

Despite the aforementioned advantages of EDGI compared to enteroscopy, EDGI carries a greater risk of AEs than enteroscopy. LAMS maldeployment and LAMS dislodgment (or spontaneous migration) in the setting of an immature gastrogastric fistula (GGF) are inherent risks of EDGl not encountered during enteroscopy. We recommend a personalized approach when selecting a primary endoscopic means of accessing the gastric remnant and duodenum in RYGB anatomy. Keeping in mind the inherent risks of EDGI, it may be preferable to use enteroscopy as the primary means for diagnosis and management of luminal pathology confined to the proximal jejunum (i. e., when seeking evaluation of the afferent limb extending from the jejunojejunal anastomosis to the ligament of Treitz). This segment of proximal jejunum is difficult to access via EDGl as it requires passage of a pediatric colonoscope through a mature GGF.

LAMS maldeployment refers to incorrect placement of the LAMS during EUS-GG (or JG), and LAMS dislodgement refers to slippage of the LAMS during transgastric (anterograde) passage of the endoscope through the LAMS. Perforation from LAMS dislodgement is only a risk while traversing an immature GGF. In a retrospective comparison of EDGE $(n=30)$ versus EA-ERCP, Bukhari et al. reported no cases LAMS maldeployment and two cases $(6.7 \%)$ of LAMS dislodgement during transgastric ERCP [16]. Both instances of LAMS dislodgement were salvaged (via LAMS repositioning and via a bridging esophageal FCSEMS), without clinical sequelae. A retrospective comparison of EDGE $(n=29)$ to LA-ERCP, by Kedia et al., reported 1 case $(3.4 \%)$ of 


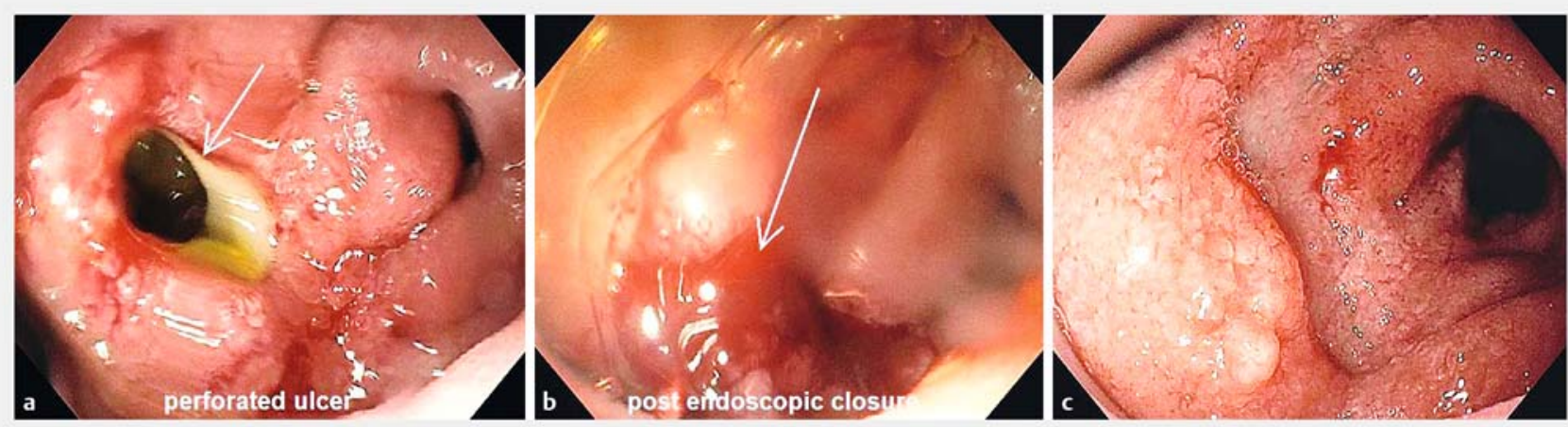

- Fig. 3 a Endoscopic view with a therapeutic gastroscope of a perforated duodenal bulb ulcer after insertion through a gastrogastric (GG) 20-mm $\times 10-\mathrm{mm}$ lumen-apposing metal stent (LAMS). $\mathbf{b}$ Endoscopic view after closure of the perforated duodenal bulb ulcer with a hemostatic clip using a therapeutic gastroscope inserted through the GG LAMS. c Endoscopic view 8 weeks after closure of the perforated duodenal bulb ulcer demonstrated healing of the ulcer. Procedure was performed using a therapeutic gastroscope inserted through the GG LAMS.

LAMS maldeployment requiring surgery and three cases (10.3\%) of LAMS dislodgment rescued with bridging esophageal FCSEMSs [18]. A retrospective EDGE study by Tyberg et al. $(n=16)$ reported four 4 cases $(25 \%)$ of stent maldeployment and/or stent dislodgement (mechanism of stent slippage is unclear) [17]. Rescue was performed via deployment of a second LAMS $(n=2)$, placement of a bridging esophageal FCSEMS $(n=$ 1 ), and perforation closure via OTSC with abortion of the intended transgastric ERCP $(n=1)$. These three retrospective EDGE studies used 15-mm (diameter) LAMS.Bukhari et al. used either freehand or over-the-wire (OTW) technique for LAMS deployment, whereas Kedia et al. and Tyberg et al. used OTW technique.

Two cases (14.3\%) of LAMS maldeployment (during EUS-GG) occurred in our study. Both instances of LAMS maldeployment occurred while using the over-the-wire (OTW) technique (i.e., stent maldeployment occurred in 2/4 [50\%] of cases using OTW technique). Freehand technique was associated with no instances of LAMS maldepolyment. Our sample size is small, but in our opinion, risk of LAMS maldeployment is likely decreased with the freehand technique due to lack of shadowing from the guidewire. The guidewire casts a sonographic shadow that can obscure the endoscopist's view of the distal flange during the initial part of LAMS deployment. We recommend adequate distention of the gastric remnant using at least 100 to $150 \mathrm{~mL}$ of contrast and sterile water, after which endosonographic deployment of the distal LAMS becomes safe under direct endosonographic visualization. OTW technique with use of ECE-LAMS also carries a theoretical risk of thermal injury and shearing of the guidewire. The benefit of the OTW technique is ease of rescue (via bridging stent) if LAMS maldeployment or LAMS dislodgment does occur.

No instances of LAMS dislodgement occurred during transgastric intervention in our five cases of single-session EDGI. It is notable that four of five cases ( $80 \%$ ) of single-session EDGI consisted of transgastric EGD. Either a diagnostic gastroscope $(n=1)$ or a therapeutic gastroscope $(n=3)$ was passed through a 20-mm LAMS after serial LAMS dilation using an 18-19-20- $\mathrm{mm}$ balloon. The exception was a case of transgastric EUS performed via single-session EDGI, in which a linear echoendoscope was passed through a 15-mm LAMS after serial LAMS dilation using a 13-14-15-mm balloon. Prior to transgastric passage of the linear echoendoscope, precaution was taken to prevent LAMS dislodgment (perforation) via LAMS fixation using two endoscopic sutures. In comparison, almost every transgastric EUS, with the exception of a single case (previously mentioned), was performed as a double procedure (i.e., 8/9 cases of transgastric EUS were performed as a dual-session EDGI).

The decision about whether to perform a single- or dual-session EDGI depends on the type of transgastric intervention (and scope type). Our opinion is that transgastric EGD at time of EUS-GG (i.e., passage of a gastroscope with 10-mm outside diameter [OD] through a freshly placed LAMS) appears safe when using a $20-\mathrm{mm}$ LAMS. In this scenario, the benefits of singlesession EDGI (i.e., cost and time savings) likely outweigh the risk of LAMS dislodgment during transgastric gastroscope passage. Passage of a linear echoendoscope (OD $14.6 \mathrm{~mm}$ ) through a newly placed $15-\mathrm{mm}$ or $20-\mathrm{mm}$ LAMS may be safe, but depends on several factors, including the angle of the placed GG LAMS and the depth that the echoendoscope must travel into the gastric remnant or duodenum to achieve the diagnostic or therapeutic intervention in question. Due to the wide outside diameter and stiff tip of the linear EUS scope, we prefer to await GGF maturation before attempting non-urgent transgastric EUS. It is our experience that a GG or JG fistula usually matures within 3 weeks of LAMS placement, after which LAMS migration during intervention does not lead to a free perforation. If single-session transgastric EUS is to be performed, the endoscopist must be vigilant. In this situation, LAMS anchoring is recommended and fluoroscopy is invaluable for avoiding acutely-angled passage of the echoendoscope through the indwelling LAMS.

Differences in total procedure time and postprocedural hospitalization were found between single-session and dual-session EDGI ( $\triangleright$ Table4). Single-session EDGI carried a lower mean procedure time $(49.4 \pm 13$ minutes vs $84.2 \pm 32.9$ min- 
utes) and a shorter median postprocedural hospitalization (0.5 days [IQR $0-1.25$ ] vs 2.5 days [IQR $0.75-11.5]$ ). The shorter procedure time of single-session EDGls is attributable to the fact that gastroduodenal luminal biopsies constituted four of five ( $80 \%$ ) single-session EDGls, compared to transgastric EUS that was performed in seven of eight $(87.5 \%)$ dual-session EDGIs. Lengthier postprocedural hospitalizations followed dual-session EDGIs because three of eight cases (37.5\%) consisted of interventional (therapeutic) procedures for acutely ill patients (i.e., closure of perforated duodenal ulcer, drainage of PFCs). No single-session EDGls were performed on acutely ill patients requiring prolonged hospitalization.

Our reported techniques for GGF closure varied by participating center ( $\triangleright$ Table 5). GGF closure via secondary intent (i.e., spontaneous closure) was most commonly used $(n=7)$, followed by application of APC to the fistula edges $(n=3)$. Routine primary GGF closure via over-the-scope clip (OTSC) or endoscopic suturing was not utilized due to concerns of cost-effectiveness. Currently, there is no unified consensus in the literature as to optimal closure of GGFs after LAMS removal. In an international, multicenter study on EDGE by Bukhari et al., $50 \%$ of patients underwent primary endoscopic fistula closure via OTSC or endoscopic suturing, and $50 \%$ of patients had deferral of primary fistula closure in favor of the possibility of spontaneous closure [16]. In one of our patient's with cholangiocarcinoma, the GGF was intentionally maintained open via a double pigtail plastic stent (i.e., DPPS placed after LAMS removal) to facilitate future pancreaticobiliary access. This patient gained $20 \mathrm{~kg}$ over the course of 1.5 years, which raises the question of whether gastric bypass reversal in the setting of malignancy is beneficial for optimizing nutritional status (i.e., to counteract the catabolic state of malignancy).

Methods of monitoring for unintentional persistent GGF also varied by participating center ( $\vee$ Table 6 ). Serial weight measurement was used in the majority of patients $(n=6)$ to monitor for unintentional weight gain (or failure to lose desired weight), which in turn would trigger ordering of an UGI series to assess for persistent GGF. Serial weight measurement is the practice of the lead institution in this study (WVU), where it is currently being studied in an IRB-approved, prospective fashion. Serial weight measurement as a means for monitoring for clinicallysignificant persistent GGF has been previously reported [16]. Other methods of surveillance for unintentional persistent GGF included UGI series $(n=3)$ and CT scan with intravenous and oral contrast $(n=1)$. Of the 10 patients who underwent monitoring for an unintentional persistent GGF in this study, none experienced unintentional weight gain (defined as more than $5 \%$ of total body weight) or had a persistent GGF diagnosed on radiographic studies. It should be noted that the follow-up period available for serial weight measurements ranged from 1 to 4 months (i. e., long-term follow-up not available given that many of the reported EDGls occurred in the months preceding the writing of this manuscript).

Limitations of our study include retrospective design, lack of a control group (e.g., no direct comparison to OAE), and small sample size $(n=14)$. Due to retrospective study design, transgastric intervention techniques varied by center, as did gastro- gastric fistula (GGF) closure technique and surveillance methods for persistent GGF. Another limitation is that four patients (28.6\%) underwent EDGE either preceding EDGI, or underwent EDGE during the same session as EDGI. These four patients were previously reported in a single-center study [9]. Reproducibility of the study will not be easy because each EDGI was performed by an expert advanced endoscopist in a tertiary center. Moreover, our definition of EDGI includes a heterogeneous collection of procedures. The definition of EDGl was made inclusive because of the relative paucity of indications.

\section{Conclusion}

In conclusion, our study demonstrated that a broad variety of EUS-directed transgastric interventions other than ERCP can be successfully performed in the excluded stomach and duodenum of RYGB patients. EDGI is a valuable addition to the procedural repertoire of advanced endoscopists because it accomplishes luminal and extraluminal interventions without reliance on percutaneous, surgical, and/or enteroscopy assistance. EDGI should be performed by advanced endoscopists with expertise in interventional EUS in high-volume tertiary care centers. We encourage multicenter collaboration for further studies on EDGI given the novelty of this procedure.

\section{Competing interests}

Dr. James receives research and training support in part by a grant from the NIH (T32DK007634). Dr. Baron is a consultant for Boston Scientific, Cook Endoscopy, W.L. Gore, and Olympus America. Dr. Khashab is a consultant for Boston Scientific, Olympus America, and Medtronic. Dr. Irani is a consultant for Boston Scientific. Dr. Nasr is a consultant for Boston Scientific.

\section{References}

[1] Buchwald H, Oien DM. Metabolic/bariatric surgery worldwide 2011. Obes Surg 2013; 23: $427-436$

[2] Tran DD, Nwokeabia ID, Purnell S. Revision of Roux-En-Y gastric bypass for weight regain: a systematic review of techniques and outcomes. Obes Surg 2016; 26: 1627 -1634

[3] Baron TH, Vickers SM. Surgical gastrostomy placement as access for diagnostic and therapeutic ERCP. Gastrointest Endosc 1998; 48: 640 641

[4] Law R, Wong Kee Song LM, Petersen BT et al. Single-session ERCP in patients with previous Roux-en-Y gastric bypass using percutaneousassisted transprosthetic endoscopic therapy: a case series. Endoscopy 2013; 45: 671-675

[5] Attam R, Leslie D, Freeman M et al. EUS-assisted, fluoroscopically guided gastrostomy tube placement in patients with Roux-en- $Y$ gastric bypass: a novel technique for access to the gastric remnant. Gastrointest Endosc 2011; 74: 677-682

[6] Kedia P, Kumta NA, Clavo CMR et al. Endoscopic ultrasound-directed transgastric ERCP (Edge) for Roux-en-Y anatomy. Gastrointest EndosC 2014; 79: AB411 
[7] Kedia P, Sharaiha RZ, Kumta NA et al. Internal EUS-directed transgastric ERCP (EDGE): game over. Gastroenterology 2014; 147: 566 - 568

[8] Kedia P, Tyberg A, Kumta NA et al. EUS-directed transgastric ERCP for Roux-en-Y gastric bypass anatomy: a minimally invasive approach. Gastrointest Endosc 2015; 82: 560 - 565

[9] James TW, Baron TH. Endoscopic ultrasound-directed transgastric ERCP (EDGE): a single-center US experience with follow-up data on fistula closure. Obes Surg 2019; 29: 451-456

[10] Cotton PB, Eisen GM, Aabakken L et al. A lexicon for endoscopic adverse events: report of an ASGE workshop. Gastrointest Endosc 2010; 71: $446-454$

[11] American Society of Metabolic and Bariatric Surgery (ASMBS). Estimate of bariatric surgery numbers. Available at: http://asmbs.org/resources/estimate-of-bariatric-surgery-numbers; Accessed December 17, 2018

[12] Kuga R, Safatle-Ribeiro AV, Faintuch J. Endoscopic findings in the excluded stomach after Roux-en-Y gastric bypass surgery. Arch Surg 2007; 142: 942 - 946

[13] Mahawar KK, Kumar P, Parmar C. Small bowel limb lengths and Rouxen-Y gastric bypass: a review. Obes Surg 2016; 26: $660-671$
[14] Siddique SS, Feuerstein JD. Gastrointestinal complications of Rouxen-Y gastric bypass surgery. OA Minim Invas Surg 2014; 2: 1

[15] Shiffman ML, Sugerman HJ, Kellum JM et al. Gallstone formation after rapid weight loss: a prospective study in patients undergoing gastric bypass surgery for treatment of morbid obesity. Am J Gastroenterol 1991; 86: $1000-1005$

[16] Bukhari M, Kowalski T, Nieto J et al. An international, multicenter, comparative trial of EUS-guided gastrogastrostomy-assisted ERCP versus enteroscopy-assisted ERCP in patients with Roux-en-Y gastric bypass anatomy. Gastrointest Endosc 2018; 88: 486-494

[17] Tyberg A, Nieto J, Salgado D et al. Endoscopic ultrasound (EUS)-directed transgastric endoscopic retrograde cholangiopancreatography or EUS: mid-term analysis of an emerging procedure. Clin Endosc 2017; 50: $185-190$

[18] Kedia P, Tarnasky PR, Nieto J et al. EUS-directed transgastric ERCP (EDGE) versus laparoscopy-assisted ERCP (LA-ERCP) for Roux-en-Y gastric bypass (RYGB) anatomy: a multicenter early comparative experience of clinical outcomes. J Clin Gastroenterol 2019; 53: 304 308 